\title{
Tenets of Community-Engaged Scholarship Applied to Delta Ways Remembered
}

\author{
Lalita Bharadwaj
}

\begin{abstract}
This essay reviews challenges posed to community-engaged scholars regarding tenure/promotion processes in Canadian universities, with a note to characteristics of community-engaged scholarship that were developed by Catherine Jordan (2007) to address gaps in academic assessment of engaged scholarship. These characteristics are: clear goals, adequate preparation, appropriate methods: scientific rigor and community engagement, significant results/impact, effective presentation/dissemination, reflective critique, leadership and personal contribution, and consistently ethical behavior. These are then applied to a non-peer reviewed work that describes the cumulative effects of environmental change for people in the Slave River Delta Region of the North West Territories, Canada. The reader is asked to view Delta Ways Remembered, a 13-minute video employing an enhanced e-storytelling technique to share and disseminate traditional knowledge about the delta from a compendium of people as a single-voiced narrative. The purpose is to highlight the scholarship underlying non-traditional academic expositions not readily assessed under current paradigms of academic evaluation. This essay strives to illustrate how Jordan's characteristics can be applied to evaluate non-peer reviewed scholarly work, and also to share rewards and challenges associated with the harmonious blending of Indigenous and western knowledge addressing societal/environmental issues identified by the Indigenous community.
\end{abstract}

KEYWORDs academic review process, Indigenous communities, non-traditional scholarly work

Community-engaged research and teaching have steadfastly gained traction within universities across Canada over the last decade. It is encouraging that promotion and support of experiential learning opportunities that may lead to community-engaged scholarship (CES) appear to be an emerging priority. However, academic institutions continue to struggle both with supporting CES and with evaluating the quality and significance of the scholarly work derived from it (Gelmon, Jordan, \& Seifer, 2013; Calleson, Jordan, \& Seifer, 2005; Saltmarsh et al., 2009). Many universities and granting agencies emphasize interdisciplinary collaborative and communityengaged scholarship as core missions (Driscoll, 2008; Sandmann, Thornton, \& Jaeger, 2009; Tierney \& Perkins, 2014) and indeed, leadership in addressing complex societal issues through various engagements with the broader community is a uniformly accepted expectation of students and faculty of Universities in Canada and internationally (Barreno et al., 2013). The adoption of CES approaches to research is generally recognized both as beneficial for higher education and as a legitimate method for knowledge generation and mobilization (Saltmarsh, 
Wooding, \& McLellan, 2014). Many universities have adapted curricula and initiated reforms to disciplinary and cross disciplinary research and educational programming towards CES. This has been observed across Canada (Barreno et al., 2013) and specific examples can be seen at the Universities of Saskatchewan, British Columbia, Victoria, and McMaster, as well as others across the nation ${ }^{1}$. For example, several universities have supported experiential learning opportunities for students through community-engaged learning funds and coursebased or one-time grants to faculty engaged in CES ${ }^{2}$.

Although such initiatives are a laudable first step to encourage faculty already disposed to CES, it does not appear they can be readily geared toward faculty who do not currently implement CES approaches, as they must step out of traditional research paradigms for exploration of these opportunities. Furthermore, it does not appear that there is adequate institutional support for crucial supplementary aspects of CES, such as research-focused community engagement or research-related knowledge translation/mobilization activities. Institutional encouragement of faculty to work in this manner, or to modify existing research programs to include such approaches, are needed at this time. Targeted financial and human resource supports for faculty/community engagements have not, as yet, been fully developed.

Mechanisms to restructure faculty workload assignments from the entrenched disciplinary assignment of duty guideline allocations of time towards teaching, research, administration, and public service are vital supports for faculty. The restructures can provide faculty with the much-needed time to effectively build essential research relationships with communities, but they are lagging. Perhaps one of the most intractable barriers has been that standards for tenure and promotion across institutions typically fail to recognize the scholarship, value, responsibilities, and roles of faculty in and with communities. The interpretation and application of standards for reviewing CES faculty through tenure and promotion processes at various university levels (departmental, college, etc.) can be difficult. In 2013, the Faculty Assessment Workgroup (members of the Community Engaged Scholarship (CES) Partnership, a research and action partnership of eight universities and one non-government organization) finalized a record of CES in tenure and promotion policy documents of sixteen Universities and three colleges in Canada (Barreno, 2013). The group reviewed the language in vision statements, in conventional institutional positioning documents, and in the professional policies, practices, and recognition of CES for the purpose of tenure and promotion. They found that most institutions lacked specific policies to address CES, although they also concluded that CES is an active and established research area in Canadian universities. Workgroup members noted that CES was fundamentally absent from institutional verbiage around tenure and promotion (Barreno, 2013).

\footnotetext{
${ }^{1}$ See University of Saskatchewan (https://www.usask.ca/engagement/), UBC (http://communityengagement.ubc.ca/), University of Victoria (https://www.uvic.ca/campusplanning/completed-projects/community-engagement/index.php), and McMaster University (https://community.mcmaster.ca/)

${ }^{2}$ See University of Saskatchewan (https://www.usask.ca/engagement/support/support.php\#Funding), and University of Victoria (https://www.uvic.ca/news/topics/2018+community-engaged-learning-grants+news; https://www.uvic.ca/news/ topics $/ 2017+$ provosts-engaged-scholars-benoit-cullen-walsh+ring
} 
Although universities typically state that they take CES into account in evaluative processes of tenure and promotion, research, and scholarship, the primary evaluation tools are overwhelmingly quantitative. Evaluative indices tend to be based predominantly on numbers of trained high-quality personnel (HQP), numbers of peer-reviewed publications, invited/ non-invited lectures and conference presentations, citations, and research grants, as well as total research funding. Although, as for all faculty, there are quantitative aspects to such evaluation criteria - for example, citations (H-index and other indices), journal quality, granting agencies etc. - these are not generally well-disposed toward an effective evaluation of CES. Faculty are more often rewarded based on traditional quantitative and qualitative evaluation protocols, but not directly on the value of scholarship to communities or other relevant contributions made towards a societal concern in which faculty have engaged in CES activities (Gelmon, Jordan, \& Seifer, 2013; Richards, 1996, Jordan et al., 2007).

Peer-reviewed publications, manuscripts, and books are the primary evidence of the impact of faculty under the category of research and scholarly work through the tenure and promotion process (Gelmon, Jordan, \& Seifer, 2013, Jordan et al., 2007). There is no argument that publications in peer-reviewed outlets are excellent forms of disseminating research information to other academics. However, they are not necessarily the most appropriate channel of dissemination with the public, with policymakers, with community leaders and their members, or with various practitioners such as social workers or health care providers (Gelmon, Jordan, \& Siefer, 2013). Thus, the rigid and rather traditional form of evaluation and interpretation of scholarly impacts in the tenure and promotion process poses structural challenges for community-engaged scholars.

Scholarship has been defined as discovery, teaching, application, integration, and engagement that has set goals, adequate preparation, appropriate methods, significant results, effective presentation, and reflective, rigourous, and peer-reviewed critique (Werber, 1992; Gelmon, Jordan, \& Siefer, 2013). Similarly, CES encompasses all these characteristics with one major distinguishing feature. People from outside the academy meaningfully and actively contribute through all aspects of the scholarship, including study goals, research design and methodology, data collections, and knowledge transfer. Although the definitions of scholarship and CES are in great alignment, CES is often misunderstood, misinterpreted, or erroneously categorized as "outreach" and/or "public service". Outreach is primarily associated with the dissemination of information and applying academic expertise to benefit external audiences. Additionally, community-engaged scholars have, in some cases, been advised to position their work with communities within the public service categories of $\mathrm{CV}$ and promotion and tenure packages (Gelmon, Jordan, \& Siefer, 2013; Driscoll, 2008) — clearly at the detriment to effective and fair evaluation of their scholarship.

After Ernest Boyer's revolutionary 1990 report, Scholarship Reconsidered: Priorities of the Professoriate, the Commission on Community-Engaged Scholarship in the Health Professions was established in 2003 to create a more supportive and rewarding system for communityengaged faculty in the United States' health professions schools. A historical step toward addressing the challenges faced by community-engaged scholars was made in the United 
States by Members of the Community-Campus Partnerships for Health (CCPH) as far back as 2007, with the development of the Community-Engaged Scholarship Review, Promotion and Tenure Package (the "Package"; Jordan, 2007). The Commission developed a set of eight characteristics, grounded in key competencies of CES and Glassick's six standards of excellence in scholarship, for the institutional evaluation of community-engaged scholarship (Glassick, Huber, \& Maeroff 1997; Jordan, 2007). These eight characteristics are:

- Clear goals,

- Adequate preparation,

- Appropriate methods: Scientific rigor and community engagement,

- Significant results/impact,

- Effective presentation/dissemination,

- Reflective critique,

- Leadership and personal contribution, and

- Consistently ethical behavior (Jordan, et al., 2007).

The CCPH recognized that there exists, generally, a gap in standards for tenure and promotion, described as an absence of any mechanisms to acknowledge unique and various forms of scholarship, its creation, and its dissemination through CES beyond that of peer-reviewed numbers and impact factors. They noted that standard definitions of a "publication" and "impact" needed to be expanded in order to include varied scholarly outputs that both are meaningful to communities and add to the academic base of knowledge (Jordan et al., 2007). They also noted that the criteria for promotion and/or tenure are lacking in defining scholarly publication and its impact, Existing criteria has tended to define these terms in modes that discount outcomes co-created by community-engaged scholars and their partners, which in turn have essential community and broader public influences (Jordan et al, 2007).

A key feature of CES is the challenge to, and opportunity for, the community-engaged scholar to generate distinct and innovative forms of scholarly works (Gelman, Jordan, $\&$ Siefer, 2013). These may include websites, crafts, documentaries, plays, manuals, briefs, posters, or other expositions. However, since these formats of scholarship are not necessarily pee- reviewed nor readily received by their peers as serious academic scholarship, there exists a structural impediment for community-engaged scholars in the academy. As the drive for CES continues within academic institutions, it becomes increasingly important that mechanisms are established for the support, evaluation, and recognition of community-engaged scholarly work, including both traditional and non-traditional outlets of scholarship.

\section{Delta Ways Remembered: A Case Study}

In order to shed some light on the nature of scholarship behind non-peer reviewed communityengaged scholarly work, in the context of the academic promotion and tenure process, this essay will reflect on a non-traditional piece of scholarly work. It will do so under Jordan's eight characteristics developed to assist in academic evaluation. The purpose is to illustrate how Jordan's characteristics could be adopted by Canadian institutions for promotion and tenure, 
as they are increasingly done so by academics in the United States.

To preface this essay, it would be helpful to view (13 minutes) the scholarly work Delta Ways Remembered ${ }^{3}$. This is a suitable example of a non-traditional piece of scholarship from the author's body of CES. While reviewing Delta Ways Remembered, I ask the reader to consider the myriad of interrelated disciplines (both academic and community disciplines) intricately woven into the scholarship. Also consider that the scholarship underwriting Delta Ways Remembered is highly interdisciplinary and thus cannot be evaluated under a single siloed discipline (art, sociology, history, drama, behavior, psychology, geography, linguistics, toxicology, environmental science, hydrology, law, journalism, etc). Review committees, at least at the departmental level, may be primarily composed of academics within the disciplinary field in which any faculty member was hired or trained, and who therefore may possess limited knowledge and understanding of CES. A concern of this type was raised by members of the CCPH who developed the set of eight characteristics within the "Package" and who recommended that institutions consider the inclusion of experienced community-engaged scholars as members of tenure and promotion review committees (Jordan et al., 2007).

Utilizing Jordan's elements, the scholarly activities involved in the creation of Delta Ways Remembered are herein explored and highlighted. The essay will reflect upon aspects of research leadership, HQP training, and the significance of publication, which are often applied criteria under which faculty are reviewed through the process of promotion and tenure in Canadian academic institutions. I begin with the first characteristic, "Clear Academic and Community Changed Goals”, and proceed through each of them sequentially.

\section{Were academic and community goals clearly defined?}

Jordan (2007) states, "The scholar must clearly define and state the objectives of his or her scholarly work and basic questions of inquiry and that goals for community change must be articulated" (p.7). Jordan goes on to describe that the evidence of clear goals involves, among other things, stating the purpose of the work, its value to the community, and identifying significant questions in the area of research.

Delta Ways Remembered was part of a Collaborative Canadian Water Network (CWN)-funded project called the Slave Watershed Environmental Effects Program (SWEEP). The goal of the SWEEP program was to co-develop a community-based cumulative effects monitoring program inclusive of Western Science (WS) and Traditional Ecological Knowledge (TEK) Indicators for the assessment of cumulative impacts on the Slave River and Delta over time. In the Slave River and Delta (SRD) watershed, changing climate and various activities in the upstream Athabasca and Peace watersheds are impacting water quantity and are potentially affecting water quality. Communities in the Northwest Territories (NWT) are concerned about the health of the SRD. In 2010, the Slave River and Delta Partnership (SRDP), representing communities along the river, was formed to address the changes people were noticing both in their environments as well as in harvested foods from the river and delta. SRDP is a collaboration of First Nations,

\footnotetext{
${ }^{3}$ https: / /www.youtube.com/watch?v=XHjmcdNwVpE
} 
Métis communities, and various agencies and organizations working, managing, and living along the Slave River. In 2011, a new partnership was formed between the SRDP, the University of Saskatchewan (USask), and the Government of Northwest Territories Environment and Natural Resources (GNWT-ENR). The partnership was to undertake an assessment of the health of the entire ecosystem. The SWEEP long-term goal was to improve the well-being of communities in the region by empowering them to monitor environmental change. Before initiating SWEEP, three guiding questions had emerged from the communities. Are the fish and wildlife safe to eat? Is the water safe to drink? Is the ecosystem healthy for our children and children's children? To help answer these community-derived questions, TEK and WS indicators of environmental change were co-created by the SRDP, USask researchers, and other community members using a "two-eyed seeing" approach (Bartlett, Marshall, \& Marshall, 2012). In collaboration with HQP (both university and community-based), we co-developed the research framework that guided the engagement, partnership building and research process to meet the goals of this project. Our collaborative group developed the TEK indicators, and eventually disseminated the Delta Ways Remembered whiteboard animated video.

\section{Was the preparation for the project adequate?}

Jordan (2007) explains that "community-engaged scholars must demonstrate that they are knowledgeable and well prepared to conduct meaningful work" (p. 77). Evidence of preparation is provided through an investment of time and effort in the discipline, and in the development of community partnerships as well as community-engaged scholarship competency.

My traditional western science background is in the fields of physiology, pathology, and toxicology. My continuing professional development in CES took place in the field over 15 years, building relationships and research partnerships with many different communities in Saskatchewan, other parts of Canada, and Peru. My CES training and research program were informed in evolutionary fashion through every community-directed project with which I engaged. I had assumed a leadership role in virtually all of these projects. The previous experience in CES, specifically with Indigenous and rural/remote communities, informed the CES activities within the Slave Watershed Environmental Effects Program program. The SWEEP program was conceived in partnership with the SRDP, and the application for funding was a collaborative effort. In the autumn of 2011, an initial introductory meeting was held in Yellowknife with the CWN program directors, members of the SRDP, and several university faculty from various institutions. This included myself and another USask faculty member who had been conducting studies of aquatic toxicity in the area. The purpose of the meeting was to provide introductions of researchers to the Slave River and Delta Partnership, and for understanding of the nature of the funding call from the CWN. This was when I first engaged with the SRDP and learned of their research priorities. A USask SWEEP team formed, and we collaborated with the established partners in the NWT to develop a project in the Slave Delta region. Our team was soon awarded funding, and our collaborative journey towards addressing the three community-derived questions began. Grounding in the communities involved the building of trust, a fragile process. This required development of familiarity not

Engaged Scholar Journal: Community-Engaged Research, Teaching and Learning 
only between a university-based researcher and the communities, but also the management of significant communication amongst communities. The scholarship behind the Delta Ways Remembered was informed by an initial project visit to the NWT in spring of 2012, followed by at least three to four week-long visits per year within the five years of the project. Attendance at a number of community events outside research activities, as well as planned workshops in member communities of the SRDP, also informed the scholarly work. The goals, research processes, and methods of data collection were informed by and evolved through an iterative process following each engagement.

\section{How methodologically-sound, rigorous, and transformative was the research?}

Scholarly work, in general, should be conducted with appropriate methods and academic rigor. Jordan (2007) states, "community-engaged scholars demonstrate that rigor is maintained or even enhanced through community-engaged approaches" (p. 77). Jordan goes on to further explain, "The involvement of the community results in scholarship that is meaningful in the real world or community setting and leads to the production of better results or in the reframing of research questions for a study" (p. 78).

The development of the research framework, the traditional ecological knowledge (TEK) indicators of the monitoring program, and the Delta Ways Remembered video could not have been envisioned or developed without the valued input from community members in the delta region. The research process blended the six faces of Traditional Ecological Knowledge with Western Science, using a two-eyed seeing approach. An understanding of the faces of TEK for inclusion in the framework and development of relevant cumulative effects indicators, could not possibly be understood from anyone else other than those with lived experiences and intergenerational knowledge. The TEK indicators that were developed and illustrated in the video were a critical requirement of the overall monitoring program as articulated by the community, and they were a major deliverable outcome of this program. The community contributions to this scholarship would not readily be appreciated as a tangible impact in the present academic review paradigm. The TEK indicators formed the basis of the communitybased cumulative effects monitoring program and clearly could not have been established without partnership and engagement with our partnering communities. Understanding of the faces of TEK was a challenge for conventionally trained academic researchers because TEK was a new concept for some and not well understood from the western scientific paradigm. The task of collectively understanding the faces of TEK, although difficult, significantly enhanced the development of the TEK indicators and the framework produced to guide the Slave River and Delta Partnership and other communities interested in conducting environmental monitoring programs ${ }^{4}$. Additionally, WS indicators ranging from identification and measurement of contaminants in the water, ice safety, and fish and wildlife health, were obtained by trained community members over a two-year period.

We refined research questions through conversations and dialogue over a two to three

\footnotetext{
${ }^{4}$ See http://cwn-rce.ca/report/slave-watershed-environmental-effects-program/
} 
year period, requiring many personal visits to these communities. Discussions took place in many venues: band offices, in the field, on boats, on rivers, in delta channels, on trap-lines, in community homes, on walks with elders along the delta shores, and in schools at elder-youth gatherings. We collectively determined the best research approach and methods to meet project objectives, and we co-designed the method for collecting information for the development of the research framework and TEK indicators (including conversation and interview methods, community asset and other forms of mapping, and historical document reviews). In addition to semi-structured interviews with land users, elders and harvesters, workshops also provided venues for data gathering. Some workshops were scheduled for other reasons outside the objectives and goals of the Slave Watershed Environmental Effects Program program, creating opportunities for further engagement and the establishment of trust and familiarity. Although these events were extremely valuable for community engagement and research rigor, arranging for attendance at these gatherings posed scheduling challenges for all involved. Information to inform the goals and the scholarly outputs that followed, including the development of the video, could not have been accomplished without the commitment of both the communities and the academic team to meaningful engagement.

As always, the success of the scholarship required engagement of the community in fiscal control and accountability aspects of the research. The team worked collaboratively with both Metis and First Nations council members and band offices in developing hiring and remuneration processes for community research assistants (CRAs) - two in this case - and in developing the scope of their employment. CRAs were trained in methods of conversation, interview, and community-concept-mapping to facilitate coordination of elder and Traditional Land User interviews. Interview guides with elders from both Metis and First Nations communities, used to elicit conversations on sensitive topics of cumulative impacts, were co-developed.

The objectives of the project were also accomplished by incorporation of historical data collected by a USask research assistant (yet to be published) from numerous historical documents about the area. Through careful application of community and methodological protocol, potential researcher biases and presuppositions were eliminated from telling the overall story, verified through community consultation and feedback from Indigenous partners.

Delta Ways Remembered is not my story, but rather it is a shared and collaborative academic work and therefore not amenable to standard forms of academic evaluation. University tenure and promotion processes still struggle to evaluate co-creative and collaborative scholarship. The philosophy behind the current tenure and promotion process is individualism.

\section{What are the project's significant results and impacts in the field and for the community?}

The community-engaged scholar must go beyond stating positive results and should demonstrate the impacts on the communityand beyond. Jordan (2007) states, "Significance of impact could include policy change, improvement of community processes or outcomes, increasing capacity of community organizations or individuals in the community to advocate for themselves, or enhancing ability of trainees to assume positions of leadership and community engagement" 
(p. 78). All these accomplishments are not readily measured and appreciated by the promotion and tenure process.

All through its inception, co-performance, dissemination, publication(s) and various outcomes, the SWEEP project has had an impact at community, University of Saskatchewan, and national levels. However, these impacts are not necessarily recognized as traditional scholarly outputs. For example, our video was featured in the Building Bridges Display, traveling to Cumberland House SK, Fort Chipewyan AB, Fort Smith NWT, Fort Resolution NWT, Yellowknife NWT, the Gordon Oakes Red Bear Student Centre at the University of Saskatchewan, and the Western Development Museum in Saskatoon. I spent significant time with this exhibit in the NWT and Alberta, travelling across the ice roads in February 2016. Building Bridges was presented to and viewed by ministers within the NWT government, and it was requested and is now part of the Montreal Science Centre exhibit on Indigenous Innovations Acting on Climate Change (IIACC). The IIACC, funded by UNESCO and the Pierre Elliot Trudeau Foundation, has travelled across Canada.

This scholarly output, in video form, has been viewed by virtually everyone in the Slave River Delta communities and has now been incorporated into the elementary and high schools curriculums of Fort Smith and Fort Resolution. It is also part of curricula at Aurora College in Fort Smith. While this type of accomplishment can be recognized by academic institutions, it takes time to come to fruition. The communities have benefited from the video because it has informed the development of a community-based cumulative effects monitoring program. It has helped to empower communities to carry out their own monitoring of cumulative impacts (CI) in the Slave River Delta. This is indeed an important and tangible example of research capacity-building at the community level. The delta communities are using the video as a tool to advocate for better CI monitoring of current and future resources and other developments. This could be recognized as impactful research, as a form of publication, and as scholarly work. It is not simply outreach.

Also noteworthy is that CES processes and outcomes are continually improved as a result of engaged scholarship. Communities have benefited from further funding and involvement in research. Most importantly, they (we) have a greater understanding, truly from an Indigenous perspective, of how the Delta has changed and how it may change in the future. It is a living perspective that one can neither obtain nor explain by flying over the region in a helicopter, by reading books, or by viewing through the lens of a single academic discipline or through the lens of a combination of western science disciplines. It took the applied CES approach to reach the Delta people's lived understandings of cumulative effects on the delta. These understandings could never be realized through traditional methods of environmental impact assessment.

Co-developed CES research skills and culturally appropriate methods of data collection/ research processes in this work were transferred to high-quality personnel at levels of community, university, and government agencies. Other University of Saskatchewan researchers

\footnotetext{
${ }^{5}$ See http://www.usask.ca/research-groups/ddn/news/index.php
} 
have recognized and are beginning to adopt these approaches. The research framework was applied, tested, and validated by a colleague and his research student for "Boreal Watershed Management Strategy Evaluating the Lake Athabasca Watershed", leading to a report prepared for the Saskatchewan Ministry of the Environment.

\section{How were project results shared with academic and community audiences?}

Jordan states, "Evidence of effective presentation might take the form of presenting at community events; publishing or broadcasting through local media; producing policy documents directed toward service providers, policy makers, or legislators as well as publishing in peer reviewed journals" (p. 78). The communication of results is a central component of what faculty do in relation to their scholarly pursuits. Communicating results, in the context of CES, must be shared with the community as well as within academia. In essence, the community-engaged scholar is expected to effectively communicate with a diversity of audiences and to possess the skills to accomplish it. Publication in peer-reviewed and/ or professional journals is an expectation, but the community-engaged scholar needs to disseminate to the communities impacted by the CES. Delta Ways Remembered can be described as academic work of CES employing an enhanced e-storytelling technique to share traditional knowledge from a compendium of people as a single-voiced narrative. This idea was gestated through conversations held with elders in Fort Resolution and Fort Smith, where they were asked: How would you like the results of this study to be shared? They answered: Not in a written report!

In October 2014, before one of my flights home from Hay River and while waiting on the tarmack, I thought of creating an RSAnimate video as a tool to effectively present and disseminate the knowledge gained by our research. Back in Saskatoon, I immediately shared this idea with my Research Associate, and thence we partnered with the University of Saskatchewan's Education Media Access Production (EMAP), as well as the Drama and Fine Arts departments (both the illustrator and the narrator were BA students). This is the first time, to the very best of my knowledge, that RSAnimate has been used to disseminate findings from a CES scholarship endeavor of this type.

In the video, a collective Indigenous voice describes knowledges of their lives - land, water and water resources, health and lifestyles, governance, stewardship, and the cumulative effects of resource development in the Slave River Delta. An accompanying peer-reviewed publication describing the methodologies associated with this scholarly work was published in the International Journal of Circumpolar Health (Bradford \& Bharadwaj, 2015). This journal was chosen with the community, as it was accessible to them.

The video was first presented to elders and council members in Fort Resolution, as part of the continuing process of verify the study findings and to consult on the effectiveness of the study dissemination. Although not a peer-reviewed publication, Delta Ways Remembered has proven to be impactful to Indigenous communities, to the Canadian public, and to the academic community.

Distribution and communication through the SWEEP program were continuous. The Slave Watershed Environmental Effects Program team met with communities and shared 
results at scheduled and non-scheduled events. For example, members of the research team communicated results at annual "Water Day" events and co-presented findings with community members at various workshops and formal academic conferences held both regionally and nationally. Videoconferences and teleconferences were also used as appropriate venues to share information. A challenge in promoting the research findings involved needing to explain technical and complex statistical results to diverse community audiences. At times, project partners had to address community disappointment and, occasionally, a disbelief in the results.

Community venues are not typically seen as academic outlets. We found texting to be an effective means of engaging with community members and for arranging sharing times. A video presentation, made to present results at a workshop event with community members, was used when academics could not attend. The video itself was shared as part of a keynote presentation at the Fourth Annual Conference NSERC CREATE H2O Centre for Human Rights Research, held at the University of Manitoba.

\section{What can be learned to improve scholarship and community engagement?}

Jordan indicates that the community-engaged scholars must possess the ability to reflect on their work critically and to assess its impact and the planning for future work meaningfully. Jordan goes on to point out that evidence of reflective critique includes holding debriefing sessions with community members or seeking their evaluation of work completed.

Formal and informal meetings with community members continued throughout the program as a forum for debriefing and evaluative opportunities of our collaborative program. Maintaining a meaningful degree of face-to-face interaction posed financial, time-related, and HQP program challenges. However, we cannot overstate the importance of this level of engagement and communication. At each meeting, community members shared their perspectives on the directions of the program, as well as the results and the opportunities for use and dissemination of the findings. For example, the symbols, words, music, and other elements of the video were all informed, modified, and finalized through conversation with community members. Due to the challenges of travel for both the academics and the community members, in-person meetings were not always feasible and telephone/video meetings were arranged. Throughout the program, four major two-day workshops were held to seek community feedback and evaluations on the research progress, methodology, and results. Although we applied a new iterative research process throughout the program, workshops were essential for summative and formative evaluation. They also provided an opportunity for more formal discussions around research processes, methods, and results. Familiarity amongst partners and the building of trust were important and valuable outcomes. A final threeday workshop was held in January 2016, to share and discuss results from SWEEP through community-researcher presentations, as well as to identify and assess outcomes and milestones from the overall project, to identify and discuss opportunities for long-term continuation of SWEEP, and to collectively discuss preferred long-term governance options for the SRDP.

With the approval of the SRDP, the video was shared with Saskatchewan Indigenous communities at several workshops, community events, and meetings. The reaction from these

Volume 5/Issue 3/Fall 2019 
communities was remarkable, and there is great interest in applying this form of dissemination in future projects. We have applied this CES/RSAnimate approach to address water and health issues with the Yellow Quill First Nation ${ }^{6}$. The various methodologies in the CES and RSAnimate have been adapted according to community needs. For example, in the co-created work with Yellow Quill First Nation, willow charcoal on heavy unbleached cotton paper was used by the artist at the community's request, and symbols were placed in accordance with the Saulteaux guiding principle of the Circle of Life. Scholarly work continues with members of the SRDP and new research questions have evolved with new funding and research programs underway.

\section{What kind of leadership and degrees of personal contribution were demonstrated by project coordinators?}

Jordan (2007) indicates that community-engaged scholars must demonstrate that "their work has earned them a reputation for rigor, impact, and the capacity to move their discipline or community change work forward and serve in a leadership role". "Evidence to demonstrate leadership and personal contribution include invited presentations (conference and community), appearance in media, external service on committees or boards" p. 79.

Leadership has been demonstrated in a number of contexts in communities across the region, with some of the impacts having been described above. In relation to CES, one of the key aspects of leadership is the ability to devolve and delegate leadership roles and responsibilities amongst partnering communities, such that the leadership is shared within the context of the research. This may be accomplished by the adoption of methodologies for Cumulative Impact Assessment. Although faculty are expected to demonstrate reputation at national and international levels through publications, grant and manuscript reviews, invited lectures, etc., one can see that the particular form of scholarly work embodied in Delta Ways Remembered does not readily conform to these categories of academic assessment. Finally, the understanding and value of traditional ecological knowledge, the very subtext of the video, is a culmination of many aspects of leadership at community and individual levels.

\section{How ethical was research and project coordination?}

Jordan indicates that project coordinators must display consistently ethical behavior. This involves the recognition and value of community knowledge systems and incorporating them into the research process. Another aspect under this characteristic is the appropriate acknowledgement of community partners when writing and presenting collaborative work.

Sensitivity to social, cultural, historical, political, and economic realities is, in my experience, one of the significant considerations for ethical and effective performance and execution of CES with (not in, for, of, or at) Indigenous communities. A program of CES, including the Delta project highlighted here, should embrace respect and understanding of community needs, protocol, and political realities. Each of these three aspects is unique in various Indigenous communities.

\footnotetext{
${ }^{6}$ See https://www.youtube.com/watch?v=NqGSm8xFR5A
} 
On the aspect of need, Indigenous communities must always identify and prioritize research direction(s) in order to effectively solve them. In the research area of Water and Health, it is important to consider that community needs extend across a vast spectrum of issues including drinking water, infectious disease, flooding, governance, environmental contamination, health services, and more. It is not ethically responsible for me, or anyone for that matter, to enter into communities with a priori established research questions/objectives and to apply them on a single or pan-community basis. This, unfortunately, is still a very common approach of university faculty interacting with Indigenous communities, and the exigencies of promotion and tenure perhaps drive it. The existing WS approach is overwhelmingly and quite understandably viewed by Indigenous people as exploitative, as it most often results in single project-based engagement with communities rather than the development of sustainable research partnerships and programs that can transform existing issues. Needs within a community are highly complex and require interdisciplinary approaches and time. A key attribute of good community-engaged scholarship is the recognition of the interrelated disciplines, and implementation of creative (co-created) approaches required to address the various community research priorities. The Delta Project illustrates this characteristic. This is not readily recognized and acknowledged as meritorious under existing academic review paradigms.

The diversity of the research outputs from a CES program should be driven by community need, remembering that the ultimate goal is to meet community needs in a meaningful way. One of the most important tenets of CES is the involvement of communities in the entire research process, including dissemination, and so very tight-knit community participation is the sine qua non of effective CES research. It is therefore not particularly amenable to high levels of peer-reviewed publication and in some cases, as discussed above, not amenable to publication in high impact factor journals. The outputs under a CES approach, nonetheless, are highly impactful.

On the aspect of protocol, there is no field guide to protocol for the various Indigenous communities in Saskatchewan, the NWT, or throughout Canada. The careful overlay of community protocol considerations with academic protocols should be a significant consideration of each and every step in CES endeavours. This extends far beyond the acquisition of Ethics Certificates at academic institutions and collaborators. Often invisible considerations of protocol must be perceived and learned on the fly, at the very first step of engagement with Indigenous or Metis communities. Many of these considerations can be imperceptible to traditional WS academics.

The very first communication is with whom? And how? Telephone, text, in-person via a bridge (such as a political or advocacy organization)? At what level of formality? It is exceedingly easy to err on all sorts of nuanced communication levels, and the results can be catastrophic. The initial communication and engagement process has been, in my experience, different in every community. Once initial engagement is established, protocol considerations must be scrupulously revisited and reflected on by the community-engaged scholar, even beyond knowledge dissemination. For example, engagement initiation and shared communication of 
research findings could variably involve presentation and dialogue with Chief and Council, sometimes with elders, sometimes with band offices, sometimes with a single councillor, sometimes with tribal councils, sometimes with schools, sometimes with all, and sometimes at the highest level of political governance within Indigenous and Metis political structures. At each step, all co-researchers need to be engaged! That's the part that is not understood by many, and the tricky part is that protocol is notoriously fluid, malleable, and ever- changing one wrong move, and everything falls apart.

In the Delta research example, it is apparent the involvement of inexperienced Masters or Ph.D.-level students (let alone post-doctoral fellows and Assistant/Associate/Full Professors) poses a risk for miscommunication. Co-workers and high-quality personnel need to spend at least a year engaging with any Indigenous community before embarking on meaningful and impactful CES. The relatively low number of university HQP inherent to this type of CES can be seen as a potential impediment to successful advancement through the ranks in academic institutions. Moreover, the timeframe for graduate program completion is often out of alignment with CES activities and community expectations, and so there is an unspoken fairness issue (on many sides) in balancing the university's academic need for student programming with the community need for deliverables.

Regarding protocol, it is incumbent upon the community-engaged scholar to consider safety, primarily as an internal institutional matter, but also for the communities with whom a scholar interacts. Consideration must be directed to safety for researchers who may, by necessity, need to work alone. In many cases, conducting the type of research underlying Delta Ways Remembered as an individual, rather than as a team, facilitates interpersonal relationships with community members. A number of safety issues were encountered in the conduction of the Slave River Delta Project, including personal isolation and geographic isolation, long time commitments to travel, elevated risks associated with rural travel modalities such as snow sleds, boat, bush plane, using rented vehicles without adequate winter tires while on ice or gravel roads, dangerous and isolated roadways subject to disruption by migratory large animal populations such as Woodland Buffalo, forest fires, and more.

Examples of community protocols to be considered and carefully managed are legion, including the selection of community research assistants, rates of CRA renumeration, rates and forms of honoraria for elders, appropriate dress code, ceremony and tradition around gifting, the manner of communication (including the use of formal and informal vernacular, humor, and eye contact), protocols of community entry, the inclusion of elders and youth, procedures around death and trauma, and other scenarios an experienced CES navigates without thinking. For the community-engaged scholar, there typically is no place in university Curriculum Vitae for the documentation of such protocols, though they are inherently positive attributes to research.

In my experience, political realities present a third level of complexity for CES work with Indigenous communities. Research must be attuned to the political landscape, and must never interfere or influence in either tangible or perceived ways. For example, funding requirements of third-party stakeholders (such as government agencies) need to be carefully and skillfully

Engaged Scholar Journal: Community-Engaged Research, Teaching and Learning 
aligned with both community (Band and Council) and university interests. Since elections are generally held every two years in Indigenous communities, changes in Chief and Council often delay research program productivity. Political changes may require re-establishment of contacts and understandings with new governance - a time consuming process. Further, as was true in the Slave River Delta, communities may be composed of members of both Metis and First Nations backgrounds, and there is a need for a nuanced balancing and equity regarding involvement of individuals in research projects, as well as in employment opportunities.

\section{Summary}

I hope to have effectively highlighted the efficacy of Jordan's eight characteristics as they may apply to the academic evaluation of even a single piece of CES; in this essay, primarily through the review of Delta Ways Remembered. They can be applied to any single work of CES disseminated in any non-traditional, non peer-reviewed format, and indeed they can be applied to entire bodies of academic work embodying the principles of CES. Significant research leadership, inter-disciplinarity, collaboration, time investment, and careful relationship-building and trust development were required to bring about production of the video. The critical issue for the community-engaged scholar is that valuable and significant outputs are not always readily amenable to dissemination in peer-reviewed outlets, nor are they always amenable to publication in what are typically considered high-impact journals. A recommendation to the CES is to craft one's justification and support for tenure and promotion around Jordan's eight characteristics. Here I make three recommendations to academic institutions regarding CES and tenure and promotion.

The first recommendation is to include community-engaged scholars in the processes of adjudication where possible. The second recommendation is to grant formal consideration to non-traditional metrics in the adjudication of tenure and promotion files, particularly for community-engaged scholars. The third recommendation is to give expanded consideration to the definition of HQP trained by community-engaged scholars (including community leaders and members). Finally, with the review of this particular scholarly work under Jordan's characteristics, I hope to have highlighted some of the challenges inherent to recognizable scholarly outputs in CES.

\section{Acknowledgements}

I would like to thank and acknowledge the people of the Slave River Delta who have shared their expertise, experience, and knowledge. I also thank Dr. Lori Bradford for her valuable contributions to Delta Ways Remembered, as well as the members of the SWEEP team. Thanks to Dr. Nicholas C. Ovsenek for his helpful comments on the manuscript. 


\section{About the Author}

Lalita Bharadwaj, Ph.D., is a Professor in the School of Public Health at the University of Saskatchewan. Email: lalita.bharadwaj@usask.ca

\section{References}

Bartlett, C., Marshall, M., Marshall, A. (2012) Two-Eyed Seeing and other lessons learned within a co-learning journey of bringing together Indigenous and mainstream knowledges and ways of knowing. Journal of Environmental Studies and Sciences, 2(4), 331-340.

Bradford, L. E., \& Bharadwaj, L. A. (2015). Whiteboard animation for knowledge mobilization: A test case from the Slave River and Delta, Canada. International Journal of Circumpolar Health, 74(1).

Barreno, L., Elliott, P.W., Madueke, I., \& Sarny, D. (2013). Research report prepared for the Faculty Assessment Workgroup Rewarding Community-Engaged Scholarship: Transforming university policies and practices. Retrieved November 30, 2019, from http:// engagedscholarship.ca/wp-content/uploads/2014/10/Faculty-Assesment-Paper1.pdf

Calleson, D.C., Jordan, C., \& Seifer, S.D. (2005). Community-engaged scholarship: Is faculty work in communities a true academic enterprise? Academic Medicine, 80, 317-321.

Driscoll, A. (2008). Carnegie's community-engagement classification: Intentions and insights. Change: The Magazine of Higher Learning, 40(1), 38-41.

Gelmon, S. B., Jordan, C., \& Seifer, S.D. (2013). Community-engaged scholarship in the Academy: An action agenda. Change: The Magazine of Higher Learning, 45(4), 58-66.

Glassick, C. E., Huber, M.T., \& Maeroff, G.I. (1997). Scholarship Assessed: Evaluation of the Professoriate. Princeton, NJ: Carnegie Foundation for the Advancement of Teaching.

Jordan, C. M., Wong, K. A., Jungnickel, P. W., Joosten, Y. A., Leugers, R. C., \& Shields, S. L. (2009). The Community-Engaged Scholarship Review, Promotion, and Tenure Package: A Guide for Faculty and Committee Members. Metropolitan Universities, 20(2), 66-86. http://depts. washington.edu/ccph/pdf_les/CES_RPT_Package.pdf

Richards, R.W. (1996). Building Partnerships: Educating Health Professionals for the Communities they Serve. San Francisco: Jossey Bass.

Saltmarsh, J., Wooding, J., \& McLellan, K. (2014). The challenges of rewarding new forms of scholarship: Creating academic cultures that support community-engaged scholarship. A report on a Bringing Theory to Practice seminar held May 15, 2014. Boston, MA: New England Resource Center for Higher Education.

Saltmarsh, J., et al. (2009). Rewarding Community-Engaged Scholarship: New Directions for Higher Education. Indianapolis, IN: Wiley.

Sandmann, L. R., Thornton, C. H., \& Jaeger, A. J. (2009). Institutionalizing Community Engagement in Higher Education: The First Wave of Carnegie Classified Institutions. San Francisco: Jossey Bass.

Tierney, W. G., \& Perkins, J. F. (2014). Beyond the ivory tower: Academic work in the 21 st century. In G. Shaker (Ed.), Faculty Work and the Public Good. New York: Teacher College.

Werber, S. (1992). On defining academic scholarship. Cleveland State Law Review, 40, 209-216. 